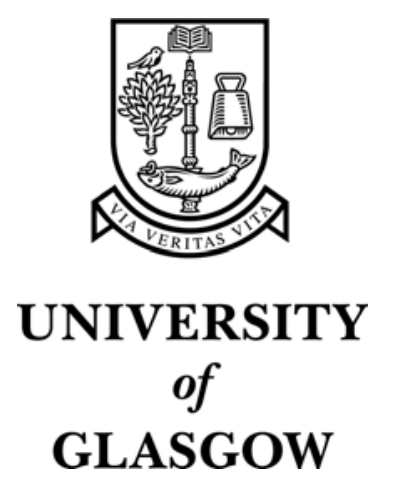

Brewster, S.A and Cryer, P.G. (1999) Maximising screen-space on mobile computing devices. In, Conference on Human Factors in Computing Systems, 15-20 May 1999, pages pp. 224-225, Pittsburgh, Pennsylvania, USA.

http://eprints.gla.ac.uk/3239/ 


\title{
Maximising Screen-Space on Mobile Computing Devices
}

\author{
Stephen A Brewster, Peter G Cryer \\ Glasgow Interactive Systems Group, Department of Computing Science, \\ University of Glasgow, Glasgow, G12 8RZ, UK.
}

Tel: +44 (0)141330 4966 / Fax: +44 (0) 1413304913 / Email: stephen@dcs.gla.ac.uk / Web: www.dcs.gla.ac.uk/ stephen/

\begin{abstract}
One major problem with mobile computing devices is lack of screen space. One way to overcome this is to reduce the size of the graphical components on screen and present information in sound. This paper describes a small pilot study to investigate the usability of sonically-enhanced buttons of different sizes. Results showed that sounds improved usability for both standard and small button sizes and that the most sophisticated sounds were the most effective.
\end{abstract}

\section{Keywords}

Earcons, mobile computers, non-speech sounds, buttons.

\section{INTRODUCTION}

Mobile computing devices are becoming extremely popular. Mobile telephones, Personal Digital Assistants (PDAs) and handheld computers are one of the fastest growth areas of computing. One problem with these devices is that there is a very limited amount of screen space: the screen cannot physically be made bigger as the devices must be able to fit into the hand or pocket to be easily carried. As the screen is small it can become cluttered with information as designers try to cram on as much as possible. This has resulted in devices that are hard to use, with small text that is hard to read, cramped graphics and little contextual information.

We suggest that sound could be used to overcome some of the problems. For example, in previous work, Brewster et al. [3, 4] used sound to improve the usability of buttons in desktop interfaces. One problem with buttons is that users can mis-press them because feedback from buttons is limited. Brewster et al. used non-speech sounds to indicate when the user was over a button, when it was pressed, when it had been released successfully and when it had been released in error. The results were good with error recovery times significantly reduced and users significantly preferring the sonically-enhanced buttons over the standard ones. These results, and others in the area [1], suggest that sound could have similar benefits on mobile computers.

One possibility would be to use sound to present information about widgets so that their size could be reduced. This would mean that the clutter on the display
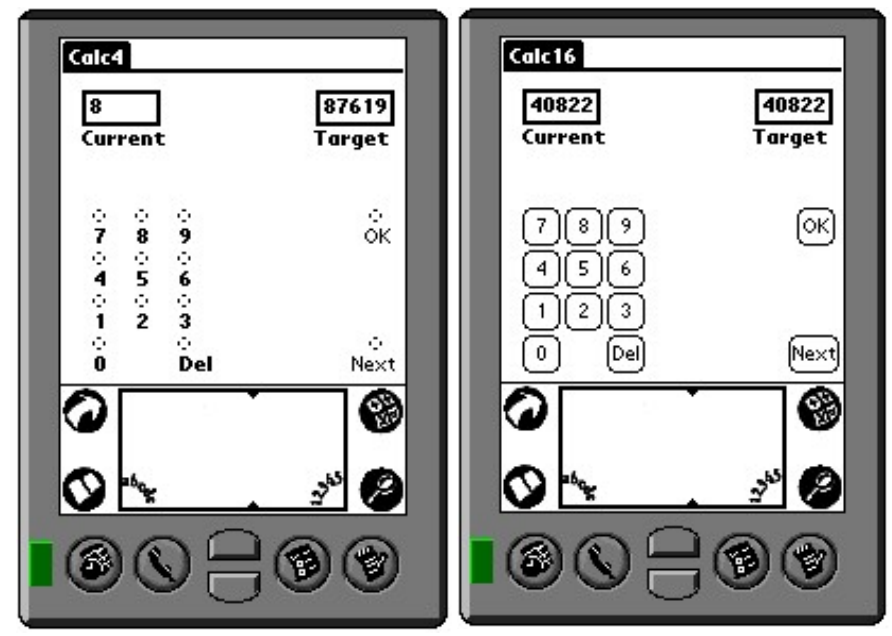

Figure 1: Screenshots of the small and standard sized buttons.

could be reduced and/or allow more information to be presented on the display. This must be done in a way that maintains usability otherwise these smaller widgets will render the device unusable. A pilot study was therefore needed to see what effect sound would have on the usability of a PDA display. On-screen buttons were chosen as these are one of the most common widgets found in the displays of mobile devices.

\begin{tabular}{|l|l|l|l|}
\hline \multicolumn{4}{|l|}{ Condition: Button size / Treatment: Sound type } \\
\hline $16 \times 16$ pixel & Silent (C1.1) & Basic sound (C1.2) & Enhanced sound (C1.3) \\
\hline $4 \times 4$ pixel & Silent (C2.1) & Basic sound (C2.2) & Enhanced sound (C2.3) \\
\hline
\end{tabular}

Table 1: Format of the pilot study.

The pilot study used a two-condition, within-groups design (see Table 1). There were two button conditions: standard size (16x16 pixels) and small (4x4 pixels). Both of these sizes are found on PDAs. There were three 7-minute treatments in each condition: silent, basic sound and enhanced sound. To measure usability workload (NASA TLX) and total amount of data entered were collected. The 12 participants used were all postgraduate students in the Department of Computing Science at Glasgow.

The experiment was run on a 3Com PalmIII handheld computer with input via a pen. The task the participants had to perform was similar to that of Brewster et al. [4]. Participants had to enter a series of five digit codes (shown in the target window in Figure 1) using the numeric keypad. After each digit had been tapped the OK button had to be pressed to confirm it. The numbers appeared in the window labelled 'Current'. Training was given before the 


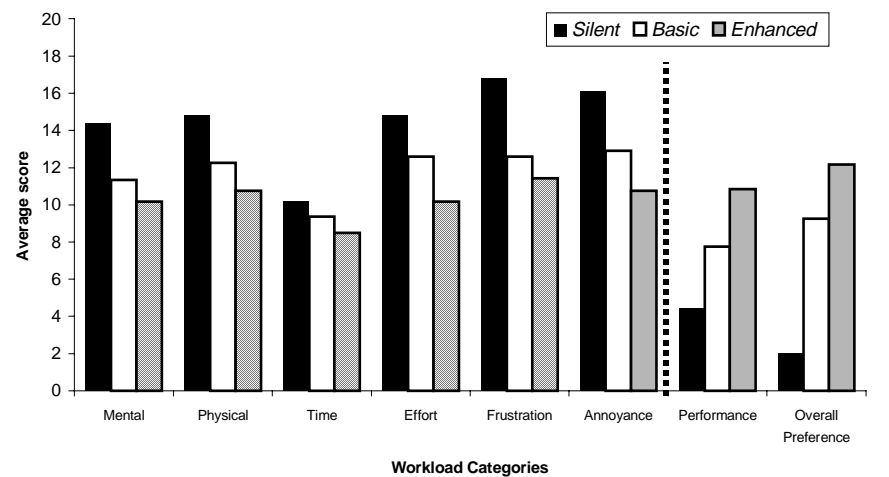

Figure 2: Average TLX scores for the small buttons. For the first 6 categories higher scores mean higher workload, for the final two (separated by the dotted line) higher scores mean lower workload.

experiment began and TLX workload charts were completed after each treatment.

Simple Earcons [2, 5] were used for the audio feedback. For the basic sounds this was just the standard keyclick sound provided on the PalmIII. This indicated that the pen was released on a button and it was selected. The enhanced sounds used the basic ones and added a sound for pen down on a button. This indicated that users had hit the target (especially important for small targets) and was a higher pitched version of the basic sound. Finally, a sound was played if the user slipped off a button [4]. It was a lower pitched version of the basic sound. The sounds were all simple as the Palm's audio capabilities are restricted.

\section{RESULTS}

For the standard buttons there was a significant reduction in overall workload $\left(F_{2,15}=15.44, \mathrm{p}=0.0002\right)$ across the three treatments. Tukey HSD tests showed that both audio treatments significantly lowered workload over the silent buttons (but no difference was shown between the two audio treatments). There was a significant overall preference for both of the audio treatments. There was a significant reduction in mental workload between the silent and the enhanced treatment only $\left(F_{2,33}=3.42, \mathrm{p}=0.004\right.$, $Q_{33}=2.58, \mathrm{p}=0.05$ ).

Figure 2 shows the results of the TLX tests for the small buttons. There was again a significant decrease in overall workload across the three treatments $\left(F_{2,33}=11.11\right.$,

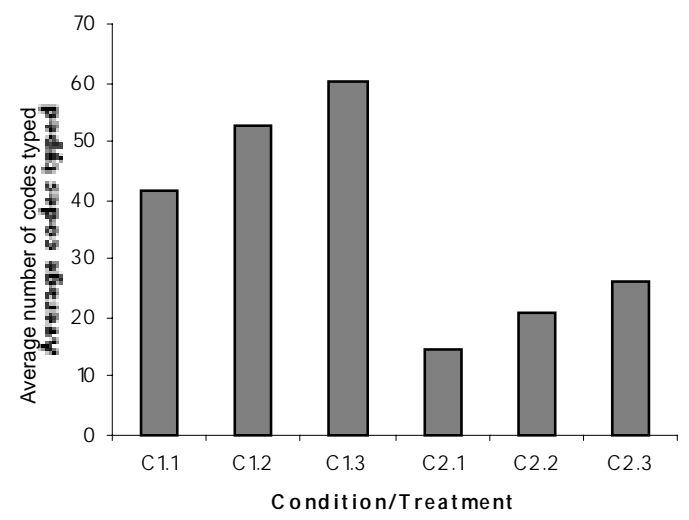

Figure 3: Average number of codes typed per condition. $\mathrm{p}=0.0001$ ). Tukey HSD tests again showed that both audio treatments significantly reduced workload. There was also a significant reduction in each of the workload categories (apart from physical demand and time pressure) for the audio treatments. In all cases, the enhanced sound was equal to or better than the basic sound. For example, mental workload was significantly reduced with the enhanced sound only $\left(F_{2,33}=5.98, \mathrm{p}=0.006, Q_{33}=4.25, \mathrm{p}=0.05\right)$. Performance was significantly higher in the enhanced sound treatment than in either the basic or silent ones $\left(F_{2,33}=16.5\right.$, $\mathrm{p}=0.00006$, enhanced vs silent: $Q_{33}=3.2, \mathrm{p}=0.05$, enhanced vs basic: $\left.Q_{33}=1.54, \mathrm{p}=0.05\right)$.

The numbers of codes typed in each condition can be seen in Figure 3. For the standard buttons there was a significant increase in the number of codes entered between both of the audio treatments and the silent one $\left(F_{2,33}=15.41\right.$, $\mathrm{p}=0.00002)$. There was no difference between the audio treatments. For the small buttons there was again an overall increase in the number of codes typed in both of the audio treatments over the silent $\left(F_{2,33}=13.53, \mathrm{p}=0.00005\right)$. A Ttest indicated that the enhanced sound significantly increased the number of codes typed over the basic sound $\left(T_{11}=5.12, \mathrm{p}=0.0003\right)$.

\section{DISCUSSION AND CONCLUSIONS}

The results of this pilot study indicate that enhancing the buttons on handheld computers with sound can significantly increase their usability. Sounds were in general useful but enhanced sounds were the most beneficial for usability, e.g. the small buttons with enhanced sound allowed significantly more data to be entered than the silent or basic sound versions. This means that designers can safely use such small buttons knowing that they will still be usable. This work also indicates that the research carried out on sound in desktop widgets [3] can be applied to hand-held devices.

\section{REFERENCES}

1. Beaudouin-Lafon, M. and Conversy, S. Auditory illusions for audio feedback. In ACM CHI'96 Conference Companion (Vancouver, Canada) ACM Press, Addison-Wesley, 1996, pp. 299-300.

2. Blattner, M., Sumikawa, D. and Greenberg, R. Earcons and icons: Their structure and common design principles. Human Computer Interaction 4, 1 (1989), 11-44.

3. Brewster, S.A. The design of sonically-enhanced widgets. Interacting with Computers (1998).

4. Brewster, S.A., Wright, P.C., Dix, A.J. and Edwards, A.D.N. The sonic enhancement of graphical buttons. In Proceedings of IFIP Interact'95 (Lillehammer, Norway) Chapman \& Hall, 1995, pp. 43-48.

5. Brewster, S.A., Wright, P.C. and Edwards, A.D.N. An evaluation of earcons for use in auditory humancomputer interfaces. In Proceedings of ACM/IFIP INTERCHI'93 (Amsterdam, Holland) ACM Press, Addison-Wesley, 1993, pp. 222-227. 